\title{
'Magic' Configurations of Three-Qubit Observables and Geometric Hyperplanes of the Smallest Split Cayley Hexagon
}

\author{
Metod SANIGA $\dagger^{\dagger^{1}}$, Michel PLANAT $\dagger^{2}$, Petr PRACNA $\dagger^{3}$ and Péter LÉVAY ${ }^{\dagger^{4}}$ \\ $\dagger^{1}$ Astronomical Institute, Slovak Academy of Sciences, \\ SK-05960 Tatranská Lomnica, Slovak Republic \\ E-mail: msaniga@astro.sk \\ $\dagger^{2}$ Institut FEMTO-ST, CNRS, 32 Avenue de l'Observatoire, F-25044 Besançon Cedex, France \\ E-mail: michel.planat@femto-st.fr \\ $\dagger^{3}$ J. Heyrovský Institute of Physical Chemistry, v.v.i., Academy of Sciences \\ of the Czech Republic, Dolejškova 3, CZ-182 23 Prague 8, Czech Republic \\ E-mail: pracna@jh-inst.cas.cz \\ $\dagger^{4}$ Department of Theoretical Physics, Institute of Physics, \\ Budapest University of Technology and Economics, H-1521 Budapest, Hungary \\ E-mail: levay@neumann.phy.bme.hu
}

Received June 22, 2012, in final form November 02, 2012; Published online November 06, 2012 http://dx.doi.org/10.3842/SIGMA.2012.083

\begin{abstract}
Recently Waegell and Aravind [J. Phys. A: Math. Theor. 45 (2012), 405301, 13 pages] have given a number of distinct sets of three-qubit observables, each furnishing a proof of the Kochen-Specker theorem. Here it is demonstrated that two of these sets/configurations, namely the $18_{2}-12_{3}$ and $22_{4} 14_{2}-4_{3} 6_{4}$ ones, can uniquely be extended into geometric hyperplanes of the split Cayley hexagon of order two, namely into those of types $\mathcal{V}_{22}(37 ; 0,12,15,10)$ and $\mathcal{V}_{4}(49 ; 0,0,21,28)$ in the classification of Frohardt and Johnson [Comm. Algebra 22 (1994), 773-797]. Moreover, employing an automorphism of order seven of the hexagon, six more replicas of either of the two configurations are obtained.
\end{abstract}

Key words: 'magic' configurations of observables; three-qubit Pauli group; split Cayley hexagon of order two

2010 Mathematics Subject Classification: 51Exx; 81R99

\section{Introduction}

For a relatively long time, the only known 'magic' configuration of three-qubit observables, that is a configuration furnishing a proof of the Kochen-Specker theorem [4], was the so-called Mermin pentagram [6] - an aggregate of ten observables forming five sets of four mutually commuting elements each, such that each observable belongs to two of these sets and the product of observables in one of them is the minus identity, whilst in the remaining four it is the plus identity. Very recently, Waegell and Aravind [19] have proposed a procedure that generates a variety of such configurations. The purpose of this note is to provide the reader, following the spirit and strategy of two recent papers [11, 12], with an intriguing finite-geometric insight into a couple of them. The relevant finite geometry is here that of the split Cayley hexagon of order two $[2,9,16]$, this being a distinguished subgeometry of the symplectic polar space $W(5,2)$ associated with the three-qubit Pauli group [3, 7, 8, 13, 14, 17], and two (out of as many as 25) distinct types of its geometric hyperplanes. 
To be more explicit, we shall make use of a highly symmetric rendering/figure [9, 16] of the split Cayley hexagon of order two, where each of its 63 points is associated with one of 63 non-trivial elements of the three-qubit Pauli group in such a way that the product of any three elements represented by the points on the same line will be proportional to the identity [5]. Then, by embedding in the hexagon (which amounts to highlighting in the figure) each of the two above-mentioned Waegell-Aravind magic configurations, we shall diagrammatically illustrate consecutive steps of 'line-completion' that in either case lead to a unique geometric hyperplane of the particular type. In addition, an automorphism of order seven of the figure in question will, in either case as well, be shown to give birth to six more configurations having the same 'magic' nature as the original one.

This short contribution is organized as follows. Section 2 highlights rudiments of the threequbit Pauli group and its associated symplectic polar space $W(5,2)$, introduces the split Cayley hexagon of order two and lists basic properties of all 25 types of its geometric hyperplanes. Section 3 deals with two particular three-qubit magic configurations of Waegell and Aravind [19] and presents a detailed demonstration of their completion into specific geometric hyperplanes of the hexagon. Finally, Section 4 summarizes main findings, mentions a parallel with the two-qubit Mermin(-Peres) magic square and outlines some prospective work.

\section{Three-qubit Pauli group and split Cayley hexagon of order two}

The (generalized) three-qubit Pauli group, $\mathcal{P}_{3}$, is generated by three-fold tensor products of the matrices

$$
I=\left(\begin{array}{ll}
1 & 0 \\
0 & 1
\end{array}\right), \quad X=\left(\begin{array}{ll}
0 & 1 \\
1 & 0
\end{array}\right), \quad Y=\left(\begin{array}{cc}
0 & -i \\
i & 0
\end{array}\right) \quad \text { and } \quad Z=\left(\begin{array}{cc}
1 & 0 \\
0 & -1
\end{array}\right) .
$$

Explicitly,

$$
\mathcal{P}_{3}=\left\{i^{\alpha} A_{1} \otimes A_{2} \otimes A_{3}: A_{j} \in\{I, X, Y, Z\}, j \in\{1,2,3\}, \alpha \in\{0,1,2,3\}\right\} .
$$

Here, we will be dealing with its factored version $\overline{\mathcal{P}}_{3} \equiv \mathcal{P}_{3} / \mathcal{Z}\left(\mathcal{P}_{3}\right)$, where the center $\mathcal{Z}\left(\mathcal{P}_{3}\right)$ consists of $\pm I \otimes I \otimes I$ and $\pm i I \otimes I \otimes I,{ }^{1}$ and whose geometry is that of the symplectic polar space $W(5,2)[3,7,8,13,14,17]$. This space, freely speaking, is a collection of all totally isotropic subspaces of the ambient five-dimensional binary projective space, $\mathrm{PG}(5,2)$, equipped with a non-degenerate alternating bilinear form. The 63 elements of the group are in a bijective correspondence with the 63 points of $W(5,2)$ in such a way that two commuting elements correspond to two points joined by a totally isotropic line; a maximum set of mutually commuting elements of the group having its counterpart in a maximal totally isotropic subspace (also called a generator), which is a projective plane of order two, the Fano plane. We shall, however, not be concerned with the full geometric structure of $W(5,2)$, but - as already mentioned - restrict ourselves to its important subgeometry represented by the split Cayley hexagon of order two. Although the two structures are identical as point-sets, the hexagon contains only 63 lines, which is much less than $W(5,2)$, and it is thus more handy to work with.

A split Cayley hexagon of order two, $\mathcal{G}_{2}$, is a point-line incidence geometry that satisfies the following axioms $[9,16]$ : a) every line contains three points and every point is contained in three lines; b) $\mathcal{G}_{2}$ does not contain any ordinary $k$-gons for $2 \leq k<6$; c) given two points, two lines, or a point and a line, there is at least one ordinary hexagon in $\mathcal{G}_{2}$ that contains both objects; and d) it contains the incidence graph of the Fano plane [1]. As $\mathcal{G}_{2}$ is rather small, it can easily be represented in a diagrammatical form, Fig. 1, from which all essential features of its geometrical structure can readily be ascertained.

\footnotetext{
${ }^{1}$ In what follows, we shall use a shorthand notation for the tensor product: $A_{1} \otimes A_{2} \otimes A_{3} \equiv A_{1} A_{2} A_{3}$.
} 


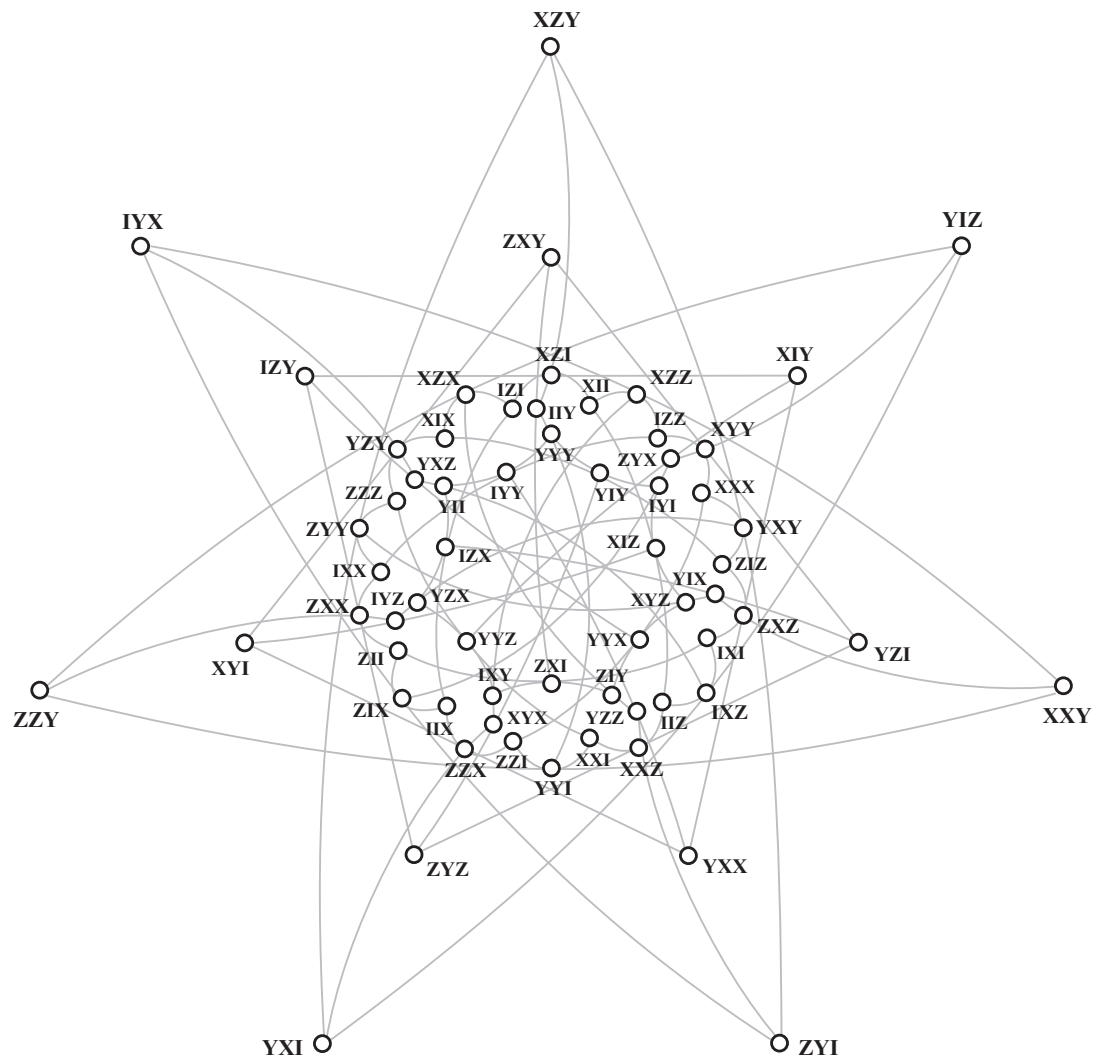

Figure 1. A diagrammatic illustration of the structure of the split Cayley hexagon of order two (based on drawings given in $[9,16])$. The points are illustrated by small circles and its lines by segments of straightlines and/or arcs; note that there are many intersections of segments that do not represent any points of the hexagon. Labeling by the elements of $\overline{\mathcal{P}}_{3}$ is adopted from [5]. Also obvious is an automorphism of order seven of the structure.

The final notion that remains to be introduced is that of a geometric hyperplane. Given any point-line incidence structure, its geometric hyperplane is a subset of the point-set such that every line of the structure either lies fully in the subset, or shares with it just one point [10]; a point of a geometric hyperplane is called deep if all the lines passing through it are fully contained in the hyperplane. It has been found [2] that $\mathcal{G}_{2}$ features $2^{14}-1=16383$ geometric hyperplanes that fall into 25 distinct types (according to the orbits of its automorphism group) and 13 classes (in terms of the sizes of their point-sets). This classification is given in Table 1, where we also adopt the Frohardt-Johnson 'five-tuple' notation [2], $\mathcal{V}_{k}\left(n ; n_{0}, n_{1}, n_{2}, n_{3}\right)$, meaning that a hyperplane of the $k$-th type, $1 \leq k \leq 25$, is endowed with $n$ points of which $n_{s}, s \in$ $\{0,1,2,3\}$, belong to exactly $s$ lines contained in the hyperplane; thus, $n_{3}$ is the number of deep points of a hyperplane. It is of some interest to note in passing that there are two distinct types of hyperplanes that have the same five-tuple, namely $\mathcal{V}_{24}$ and $\mathcal{V}_{25}$

At this point we have introduced all the necessary finite-geometrical technicalities to be employed in the next section to analyse some 'magic' configurations of three-qubit observables.

\section{Waegell-Aravind configurations uniquely extendible into geometric hyperplanes}

We shall first deal with the configuration shown in Fig. 5 of [19], bearing there symbol $18_{2}-12_{3}$. It consists of 18 observables forming 12 sets of mutually commuting elements of size three each, 
Table 1. Classes and types of geometric hyperplanes of the split Cayley hexagon of order two. For each type one gives the size of its point- ('Pts') and line- ('Lns') sets, number of deep points ('DPts'), total number of distinct copies ('Cps') and the stabilizer group ('StGr') of its orbit; for more group-theoretical details, see [2].

\begin{tabular}{||l|l|rrrrl||}
\hline \hline Class & FJ Type & Pts & Lns & DPts & Cps & StGr \\
\hline \hline I & $\mathcal{V}_{2}(21 ; 21,0,0,0)$ & 21 & 0 & 0 & 36 & $P G L(2,7)$ \\
\hline II & $\mathcal{V}_{7}(23 ; 16,6,0,1)$ & 23 & 3 & 1 & 126 & $(4 \times 4): S_{3}$ \\
\hline III & $\mathcal{V}_{11}(25 ; 10,12,3,0)$ & 25 & 6 & 0 & 504 & $S_{4}$ \\
\hline IV & $\mathcal{V}_{1}(27 ; 0,27,0,0)$ & 27 & 9 & 0 & 28 & $X_{27}^{+}: Q D_{16}$ \\
& $\mathcal{V}_{8}(27 ; 8,15,0,4)$ & 27 & 9 & $3+1$ & 252 & $2 \times S_{4}$ \\
& $\mathcal{V}_{13}(27 ; 8,11,8,0)$ & 27 & $8+1$ & 0 & 756 & $D_{16}$ \\
& $\mathcal{V}_{17}(27 ; 6,15,6,0)$ & 27 & $6+3$ & 0 & 1008 & $D_{12}$ \\
\hline V & $\mathcal{V}_{12}(29 ; 7,12,6,4)$ & 29 & 12 & 4 & 504 & $S_{4}$ \\
& $\mathcal{V}_{18}(29 ; 5,12,12,0)$ & 29 & 12 & 0 & 1008 & $D_{12}$ \\
& $\mathcal{V}_{19}(29 ; 6,12,9,2)$ & 29 & 12 & $2 n c$ & 1008 & $D_{12}$ \\
& $\mathcal{V}_{23}(29 ; 4,16,7,2)$ & 29 & 12 & $2 \mathrm{c}$ & 1512 & $D_{8}$ \\
\hline VI & $\mathcal{V}_{6}(31 ; 0,24,0,7)$ & 31 & 15 & $6+1$ & 63 & $(4 \times 4): D_{12}$ \\
& $\mathcal{V}_{24}(31 ; 4,12,12,3)$ & 31 & 15 & $2+1$ & 1512 & $D_{8}$ \\
& $\mathcal{V}_{25}(31 ; 4,12,12,3)$ & 31 & 15 & 3 & 2016 & $S_{3}$ \\
\hline VII & $\mathcal{V}_{14}(33 ; 4,8,17,4)$ & 33 & 18 & $2+2$ & 756 & $D_{16}$ \\
& $\mathcal{V}_{20}(33 ; 2,12,15,4)$ & 33 & 18 & $3+1$ & 1008 & $D_{12}$ \\
\hline VIII & $\mathcal{V}_{3}(35 ; 0,21,0,14)$ & 35 & 21 & 14 & 36 & $P G L(2,7)$ \\
& $\mathcal{V}_{16}(35 ; 0,13,16,6)$ & 35 & 21 & $4+2$ & 756 & $D_{16}$ \\
& $\mathcal{V}_{21}(35 ; 2,9,18,6)$ & 35 & 21 & 6 & 1008 & $D_{12}$ \\
\hline IX & $\mathcal{V}_{15}(37 ; 1,8,20,8)$ & 37 & 24 & 8 & 756 & $D_{16}$ \\
& $\mathcal{V}_{22}(37 ; 0,12,15,10)$ & 37 & 24 & $6+3+1$ & 1008 & $D_{12}$ \\
\hline X & $\mathcal{V}_{10}(39 ; 0,10,16,13)$ & 39 & 27 & $8+4+1$ & 378 & $8: 2: 2$ \\
\hline XI & $\mathcal{V}_{9}(43 ; 0,3,24,16)$ & 43 & 33 & $12+3+1$ & 252 & $2 \times S_{4}$ \\
\hline XII & $\mathcal{V}_{5}(45 ; 0,0,27,18)$ & 45 & 36 & 18 & 56 & $X_{27}^{+}: D_{8}$ \\
\hline XIII & $\mathcal{V}_{4}(49 ; 0,0,21,28)$ & 49 & 42 & 28 & 36 & $P G L(2,7)$ \\
\hline \hline & & & & & & \\
\hline
\end{tabular}

namely:

$$
\begin{array}{lll}
\{I Z I, Z Z I, Z I I\}, & \{Z I I, Z I Z, I I Z\}, & \{I I Z, I Z Z, I Z I\}, \\
\{I X I, X X I, X I I\}, & \{X I I, X I X, I I X\}, & \{I I X, I X X, I X I\}, \\
\{I Y I, Y Y I, Y I I\}, & \{Y I I, Y I Y, I I Y\}, & \{I I Y, I Y Y, I Y I\}, \\
\{Z Z I, X X I, Y Y I\}, & \{Y I Y, X I X, Z I Z\}, & \{I Z Z, I X X, I Y Y\},
\end{array}
$$

each of which represents a line in $W(5,2)$. It is readily verified that the product of observables in each set is $+I I I$ except for the last three sets where it is $-I I I$. Given this fact and the fact that each observable belongs to exactly two sets, it is impossible to assign the eigenvalue +1 or -1 to each observable in such a way that these obey the same product rules as the observables themselves - this rendering a proof of the Kochen-Specker theorem [19].

It is, however, not this theorem that is of concern for us here. Rather, it is the configuration as a whole and, in particular, its image within our split Cayley hexagon, as depicted in Fig. 2, left, by red bullets. Let us try to find a geometric hyperplane this configuration sits in. To this end, one has simply to recall that a line of the hexagon is either fully contained in a hyperplane, or shares with it a single point. A brief look at Fig. 2, left, reveals that there are a number of 

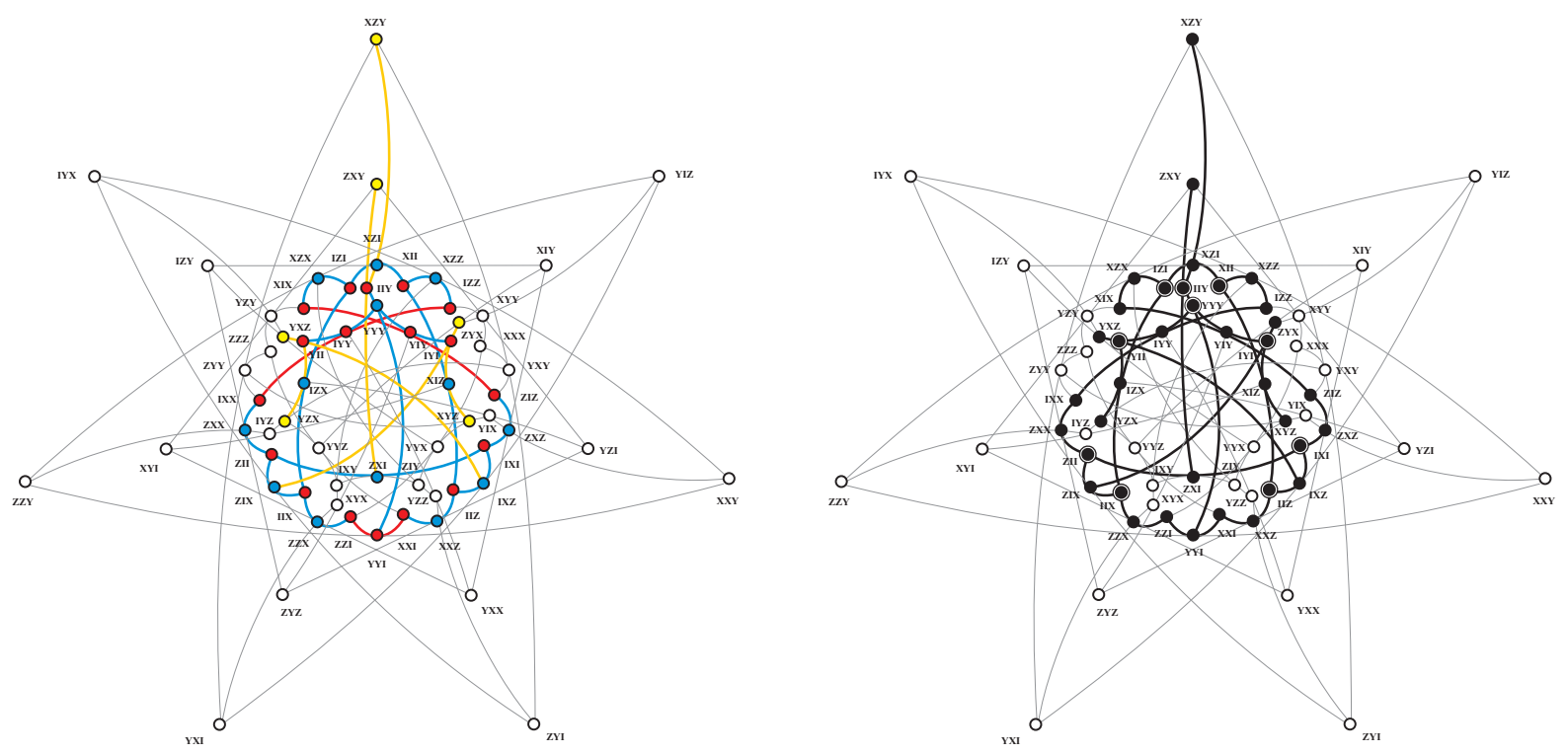

Figure 2. Left: An illustration of the process of extension of the set of three-qubit observables of the $18_{2}-12_{3}$ magic configuration of Waegell and Aravind [19, Fig. 5] into a geometric hyperplane of the split Cayley hexagon of order two. Red bullets are the points/observables of the configuration itself, blue bullets represent the remaining points on the lines of the hexagon featuring two red points and yellow bullets stand for the remaining points on the lines of the hexagon having one red and one blue point. Right: A simplified illustration of the structure of the corresponding hyperplane; encircled are all the ten deep points of the hyperplane.

lines of the hexagon that contains two red points and, hence, must lie completely within our sought-for hyperplane; any such line, as well as the remaining third point on it, is illustrated in blue. By extending our original configuration by the blue points and lines, we shall find some lines to feature one red and one blue point; these are highlighted in yellow and must equally belong fully to the sought-for hyperplane. At this step our process of extension ends, for this doubly-extended aggregate of points, illustrated in a colorless version in Fig. 2, right, already satisfies the properties of a geometric hyperplane. From this figure we easily discern that our hyperplane has 37 points and 10 deep points, and from Table 1 we find out that it must be of type $\mathcal{V}_{22}(37 ; 0,12,15,10)$, since the only other type of the same size has, for example, only 8 deep points.

The (only) other W-A configuration that is subject to a unique extension into a geometric hyperplane is the $2{ }_{4} 14_{2}-4_{3} 6_{4}$ one, [19, Fig. 8 , right], comprising the following sets of pairwise commuting observables:

$$
\begin{array}{lll}
\{Z I Z, Z I I, I I Z\}, & \{I I Z, I Z I, I Z Z\}, \\
\{Z I I, I Z I, Z Z I\}, & \{I Z Z, X Y Y, X X X\}, \\
\{I I X, Y I I, I Y I, Y Y X\}, & \{I Y I, I I Y, X I I, X Y Y\}, \\
\{Y I I, I I Y, I X I, Y X Y\}, & \{I I X, X I I, I X I, X X X\}, \\
\{I Z Z, Z Z I, Y X Y, X X X\}, & \{I Z Z, Z I Z, Y Y X, X X X\} .
\end{array}
$$

Geometrically speaking, each three-element set corresponds to a line of $W(5,2)$, whereas each four-element set represents an affine plane of order two (located in a certain Fano plane) of $W(5,2)$. Again, we are interested in the configuration as a whole and highlighting this set of observables in the figure of the hexagon and following the same strategy as in the preceding case we shall find that this configuration is uniquely extendible into a geometric hyperplane of 

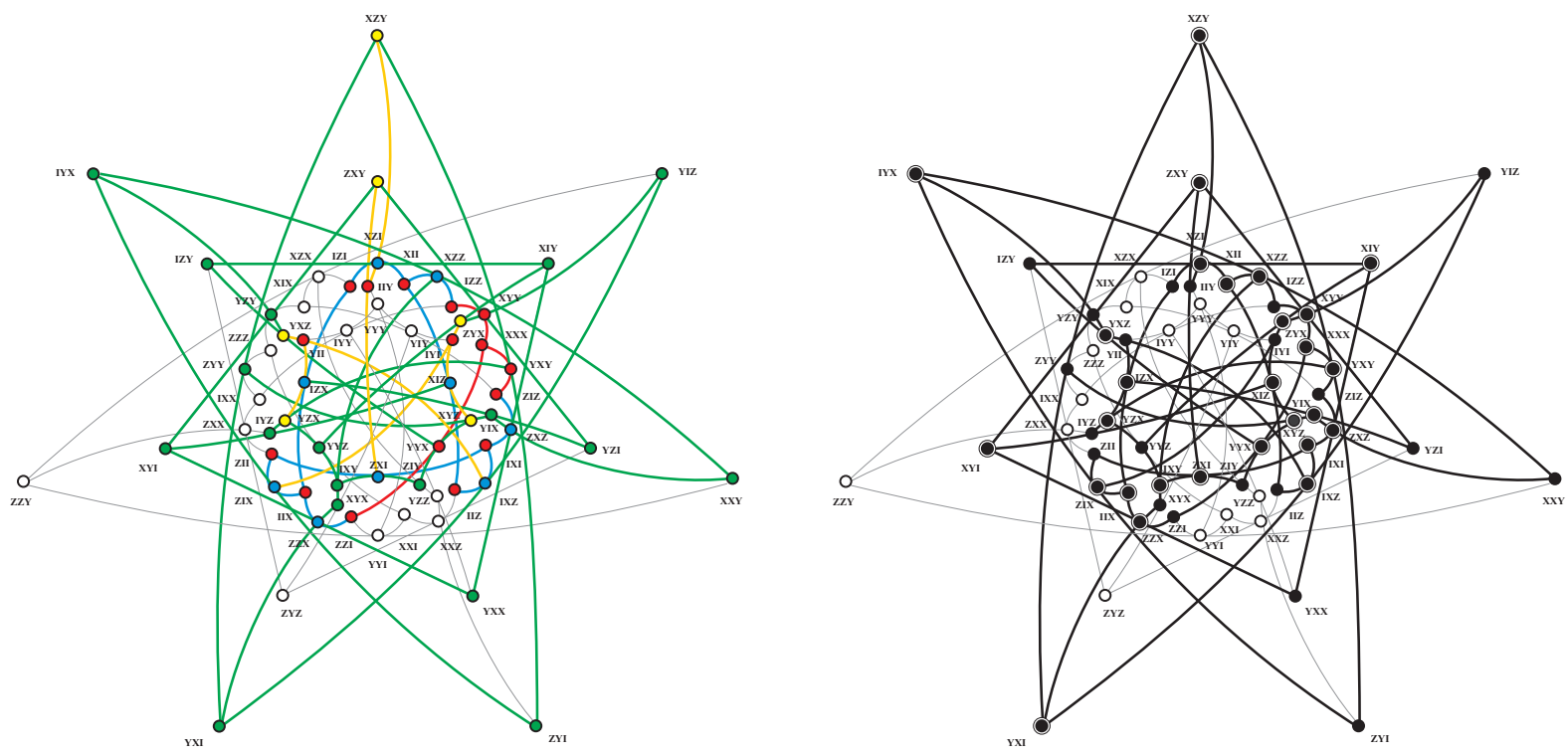

Figure 3. Left: The same as in Fig. 2 for the $2_{4} 14_{2}-4_{3} 6_{4}$ 'magic' configuration. The meaning of colors is also the same as in the preceding figure save for the fact that we now need a few more steps (for simplicity all illustrated by green color) to arrive at a hyperplane; in particular, a green point is the third point on the line of the hexagon that features either a red point and a yellow one, or a blue/yellow point and a(n already supplied in a previous step) green one. Right: A simplified sketch of the resulting hyperplane with its deep points encircled.

type $\mathcal{V}_{4}(49 ; 0,0,21,28)$ - see Fig. 3. A hyperplane of this type, apart from being of the largest possible size (see Table 1), is also remarkable by the fact that its complement is nothing but the incidence graph of the Fano plane.

As for the remaining W-A configurations, none of them was found to be uniquely extendible into a geometric hyperplane. Except for the $1_{4} 11_{2}-2{ }_{3} 5_{4}$ configuration [19, Fig. 8, left], which does not seem to be embeddable into any hyperplane, all the other configurations were found to sit in at least two distinct types of hyperplanes; in particular, the three-qubit Peres-Mermin square [19, Fig. 4] was found in as many as 12 different types of hyperplanes.

We shall conclude this section by observing that an automorphism of order seven of our hexagon can be used to generate six more replicas (listed below in columns $b$ to $g$ ) for either of the above discussed W-A configurations (column $a$ ). Technically, this action is performed by rotating consecutively Fig. 2, left, and Fig. 3, left, by 360/7 degrees around the center of the hexagon. Thus, rotating the figures in question counterclockwise, the interested reader can easily check that for the $18_{2}-12_{3}$ case we get

\begin{tabular}{|c|c|c|c|c|c|c|c|c|c|c|c|}
\hline$a$ & $\mapsto$ & $b$ & $\mapsto$ & $c$ & $\mapsto$ & $d$ & $\mapsto$ & $e$ & $\mapsto$ & $f$ & $\mapsto$ \\
\hline$X I X$ & $\mapsto$ & $I X X$ & $\mapsto$ & $I I X$ & $\mapsto$ & $X X I$ & $\mapsto$ & $I X I$ & $\mapsto$ & $X X X$ & $\mapsto X I I$ \\
\hline$I Z I$ & $\mapsto$ & $Z Z Z$ & $\mapsto$ & $Z I I$ & $\mapsto$ & $Z Z I$ & $\mapsto$ & $I I Z$ & $\mapsto$ & $Z I Z$ & $I Z Z$ \\
\hline$X I I$ & $\mapsto$ & $X I X$ & $\mapsto$ & $I X X$ & $\mapsto$ & $I I X$ & $\mapsto$ & $X X I$ & $\mapsto$ & $I X I$ & $\mapsto X X X$ \\
\hline$I Z Z$ & $\mapsto$ & $I Z I$ & $\mapsto$ & $Z Z Z$ & $\mapsto$ & $Z I I$ & $\mapsto$ & $Z Z I$ & $\mapsto$ & $I I Z$ & $\mapsto Z I Z$ \\
\hline$Y I I$ & $\mapsto$ & $Y Z X$ & $\mapsto$ & $I X Y$ & $\mapsto$ & $Z I Y$ & $\mapsto$ & $X Y Z$ & $\mapsto$ & $I Y I$ & $\mapsto Y Y Y$ \\
\hline$I Y Y$ & $\mapsto$ & $I Z X$ & $\mapsto$ & $Y Y Z$ & $\mapsto$ & $Z X I$ & $\mapsto$ & $Y Y X$ & $\mapsto$ & $X I Z$ & $\mapsto Y I Y$ \\
\hline$Y I Y$ & $\mapsto$ & $I Y Y$ & $\mapsto$ & $I Z X$ & $\mapsto$ & $Y Y Z$ & $\mapsto$ & $Z X I$ & $\mapsto$ & $Y Y X$ & $X I Z$ \\
\hline$I Y I$ & $\mapsto$ & $Y Y Y$ & $\mapsto$ & $Y I I$ & $\mapsto$ & $Y Z X$ & $\mapsto$ & $I X Y$ & $\mapsto$ & $Z I Y$ & $X Y Z$ \\
\hline$I X X$ & $\mapsto$ & $I I X$ & $\mapsto$ & $X X I$ & $\mapsto$ & $I X I$ & $\mapsto$ & $X X X$ & $\mapsto$ & $X I I$ & $X I X$ \\
\hline
\end{tabular}




$\begin{array}{llllllll}Z I I & \mapsto Z Z I & \mapsto I I Z & \mapsto Z I Z & \mapsto I Z Z & \mapsto I Z I & \mapsto Z Z Z, \\ I I X & \mapsto X X I & \mapsto I X I & \mapsto X X X & \mapsto X I I & \mapsto X I X & \mapsto I X X, \\ Z Z I & \mapsto I I Z & \mapsto Z I Z & \mapsto I Z Z & \mapsto I Z I & \mapsto Z Z Z & \mapsto Z I I, \\ Y Y I & \mapsto I X Z & \mapsto Y X Y & \mapsto X Z Z & \mapsto X Z X & \mapsto Z Y Y & \mapsto Z I X, \\ X X I & \mapsto I X I & \mapsto X X X & \mapsto X I I & \mapsto X I X & \mapsto I X X & \mapsto I I X, \\ I I Z & \mapsto Z I Z & \mapsto I Z Z & \mapsto I Z I & \mapsto Z Z Z & \mapsto Z I I & \mapsto Z Z I, \\ I X I & \mapsto X X X & \mapsto X I I & \mapsto X I X & \mapsto I X X & \mapsto I I X & \mapsto X X I, \\ Z I Z & \mapsto I Z Z & \mapsto I Z I & \mapsto Z Z Z & \mapsto Z I I & \mapsto Z Z I & \mapsto I I Z, \\ I I Y & \mapsto Y X Z & \mapsto I Y Z & \mapsto X Y X & \mapsto Y Z Z & \mapsto Y I X & \mapsto Z Y X,\end{array}$

and for the $22_{4} 14_{2}-4_{3} 6_{4}$ case we obtain

$\begin{array}{llllllllll}a & \mapsto b & \mapsto & \mapsto & \mapsto & \mapsto & b & \mapsto & f & \mapsto \\ I Z I & \mapsto Z Z Z & \mapsto Z I I & \mapsto Z Z I & \mapsto I I Z & \mapsto Z I Z & \mapsto I Z Z, \\ I I Y & \mapsto X X Z & \mapsto I Y Z & \mapsto X Y X & \mapsto Y Z Z & \mapsto Y I X & \mapsto Z Y X, \\ X I I & \mapsto X I X & \mapsto I X X & \mapsto I I X & \mapsto X X I & \mapsto I X I & \mapsto X X X, \\ I Z Z & \mapsto I Z I & \mapsto Z Z Z & \mapsto Z I I & \mapsto Z Z I & \mapsto I I Z & \mapsto Z I Z, \\ X Y Y & \mapsto X Z I & \mapsto Y Z Y & \mapsto Z X X & \mapsto Z Z X & \mapsto X X Z & \mapsto Z X Z, \\ X X X & \mapsto X I I & \mapsto X I X & \mapsto I X X & \mapsto I I X & \mapsto X X I & \mapsto I X I, \\ Y X Y & \mapsto X Z Z & \mapsto X Z X & \mapsto Z Y Y & \mapsto Z I X & \mapsto Y Y I & \mapsto I X Z, \\ Z I Z & \mapsto I Z Z & \mapsto I Z I & \mapsto Z Z Z & \mapsto Z I I & \mapsto Z Z I & \mapsto I I Z, \\ I X I & \mapsto X X X & \mapsto X I I & \mapsto X I X & \mapsto I X X & \mapsto I I X & \mapsto X X I, \\ I I Z & \mapsto Z I Z & \mapsto I Z Z & \mapsto I Z I & \mapsto Z Z Z & \mapsto Z I I & \mapsto Z Z I, \\ Z Z I & \mapsto I I Z & \mapsto Z I Z & \mapsto I Z Z & \mapsto I Z I & \mapsto Z Z Z & \mapsto Z I I, \\ I I X & \mapsto X X I & \mapsto I X I & \mapsto X X X & \mapsto X I I & \mapsto X I X & \mapsto I X X, \\ Z I I & \mapsto Z Z I & \mapsto I I Z & \mapsto Z I Z & \mapsto I Z Z & \mapsto I Z I & \mapsto Z Z Z, \\ Y I I & \mapsto Y Z X & \mapsto I X Y & \mapsto Z I Y & \mapsto X Y Z & \mapsto I Y I & \mapsto Y Y Y, \\ I Y I & \mapsto Y Y Y & \mapsto Y I I & \mapsto Y Z X & \mapsto I X Y & \mapsto Z I Y & \mapsto X Y Z, \\ Y Y X & \mapsto X I Z & \mapsto Y I Y & \mapsto I Y Y & \mapsto I Z X & \mapsto Y Y Z & \mapsto Z X I\end{array}$

We have also verified that all these replicas are 'magic' in the same way as the configuration we started with, and each of them can thus be used as a proof of the KS theorem. Similarly, we can get six magic replicas for any other W-A configuration by embedding it into the hexagon and 'acting' on it by the automorphism in question.

\section{Conclusion}

Employing the structure of the split Cayley hexagon of order two, the smallest non-trivial generalized hexagon and a distinguished subgeometry of the symplectic polar space $W(5,2)$ of the three-qubit Pauli group, we got an intriguing finite-geometric insight into the nature of a couple of 'magic' three-qubit configurations proposed recently by Waegell and Aravind [19], namely the $18_{2}-12_{3}$ and $2{ }_{4} 14_{2}-4_{3} 6_{4}$ ones. Either of the two configurations was found to be uniquely extendible into a geometric hyperplane of the hexagon, this being, respectively, of type $\mathcal{V}_{22}(37 ; 0,12,15,10)$ and $\mathcal{V}_{4}(49 ; 0,0,21,28)$ in the classification of Frohardt and Johnson [2]. Moreover, an automorphism of order seven of the hexagon gave birth, for either of the two, to six more replicas, each having the same 'magical' nature as the parent one. 
It is important to emphasize that this is, to our best knowledge, only the second instance where geometric hyperplanes are related to certain 'magic' configurations of observables. The first instance was the Mermin square of two-qubits, which was recognized to be a full geometric hyperplane of the generalized quadrangle of order two, $\operatorname{GQ}(2,2)$ (see, e.g., $[7,8,14]$ ). However, in a more general setting of quantum information theory, geometric hyperplanes have already entered the game through the concept of a Veldkamp space $[15,18]$. Last but not least, we have to mention that this smallest split Cayley hexagon has also been found to play a role in the closely-related context of the so-called black-hole-qubit correspondence [5]; here also one of its geometric hyperplanes, the so-called distance-2-ovoid (of type $\mathcal{V}_{2}(21 ; 21,0,0,0)$ ), was mentioned in connection with a certain class of quantum codes.

That there is more behind this smallest split Cayley hexagon than meets the eye is also indicated by the following observation. One of the most symmetric, and most pronounced as well, three-qubit 'magic' configurations is the so-called Mermin pentagram (see, e.g., [6, 11, 19]). Using computer, we have found that the full symplectic geometry of the three-qubit Pauli group, $W(5,2)$ contains altogether 12096 copies of such a pentagram, this number being - remarkably equal to the order of the automorphism group of our hexagon.

In their paper [19, p. 7], Waegell and Aravind stress that "we have not displayed all the diagrams we have found, but only a representative sample". It would certainly be of great interest for us to become familiar with those yet unpublished and analyze them in the abovedescribed fashion in order to see whether there is some underlying link between those that can uniquely be extended into a geometric hyperplane of our hexagon. We would, eventually, be most curious to see if each type of a geometric hyperplane can serve as a unique extension of some 'magic' configuration(s), or whether this is a privilege for only some of them.

\section{Acknowledgement}

This work was partially supported by the VEGA grant agency project 2/0098/10.

\section{References}

[1] De Kaey J., Van Maldeghem H., A characterization of the split Cayley generalized hexagon $H(q)$ using one subhexagon of order (1,q), Discrete Math. 294 (2005), 109-118.

[2] Frohardt D., Johnson P., Geometric hyperplanes in generalized hexagons of order (2, 2), Comm. Algebra 22 (1994), 773-797.

[3] Havlicek H., Odehnal B., Saniga M., Factor-group-generated polar spaces and (multi-)qudits, SIGMA 5 (2009), 096, 15 pages, arXiv:0903.5418.

[4] Kochen S., Specker E.P., The problem of hidden variables in quantum mechanics, J. Math. Mech. 17 (1967), 59-87.

[5] Lévay P., Saniga M., Vrana P., Three-qubit operators, the split Cayley hexagon of order two, and black holes, Phys. Rev. D 78 (2008), 124022, 16 pages, arXiv:0808.3849.

[6] Mermin N.D., Hidden variables and the two theorems of John Bell, Rev. Modern Phys. 65 (1993), 803-815.

[7] Planat M., Pauli graphs when the Hilbert space dimension contains a square: why the Dedekind psi function?, J. Phys. A: Math. Theor. 44 (2011), 045301, 16 pages, arXiv:1009.3858.

[8] Planat M., Saniga M., On the Pauli graphs on N-qudits, Quantum Inf. Comput. 8 (2008), 127-146, quant-ph/0701211.

[9] Polster B., Schroth A.E., van Maldeghem H., Generalized flatland, Math. Intelligencer 23 (2001), 33-47.

[10] Ronan M.A., Embeddings and hyperplanes of discrete geometries, European J. Combin. 8 (1987), 179-185.

[11] Saniga M., Lévay P., Mermin's pentagram as an ovoid of PG(3,2), Europhys. Lett. 97 (2012), 50006,3 pages, arXiv:1111.5923.

[12] Saniga M., Planat M., Finite geometry behind the Harvey-Chryssanthacopoulos four-qubit magic rectangle, Quantum Inf. Comput. 11 (2012), 1011-1016, arXiv:1204.6229. 
[13] Saniga M., Planat M., Multiple qubits as symplectic polar spaces of order two, Adv. Stud. Theor. Phys. 1 (2007), 1-4, quant-ph/0612179.

[14] Saniga M., Planat M., Prachna P., Projective curves over a ring that includes two-qubits, Theoret. and Math. Phys. 155 (2008), 905-913, quant-ph/0611063.

[15] Saniga M., Planat M., Pracna P., Havlicek H., The Veldkamp space of two-qubits, SIGMA 3 (2007), 075, 7 pages, arXiv:0704.0495.

[16] Schroth A.E., How to draw a hexagon, Discrete Math. 199 (1999), 161-171.

[17] Thas K., The geometry of generalized Pauli operators of $N$-qudit Hilbert space, and an application to MUBs, Europhys. Lett. 86 (2009), 60005, 3 pages.

[18] Vrana P., Lévay P., The Veldkamp space of multiple qubits, J. Phys. A: Math. Theor. 43 (2010), 125303, 16 pages, arXiv:0906.3655.

[19] Waegell M., Aravind P.K., Proofs of the Kochen-Specker theorem based on a system of three qubits, J. Phys. A: Math. Theor. 45 (2012), 405301, 13 pages, arXiv:1205.5015. 